\title{
JNK Isoforms Differentially Regulate Neurite Growth and Regeneration in Dopaminergic Neurons In Vitro
}

\author{
Lars Tönges • Veronique Planchamp • \\ Jan-Christoph Koch • Thomas Herdegen • \\ Mathias Bähr • Paul Lingor
}

Received: 19 January 2011 / Accepted: 24 March 2011 / Published online: 6 April 2011

(C) The Author(s) 2011. This article is published with open access at Springerlink.com

\begin{abstract}
Parkinson's disease is characterized by selective and progressive loss of midbrain DAergic neurons (MDN) in the substantia nigra and degeneration of its nigrostriatal projections. Whereas the cellular pathophysiology has been closely linked to an activation of c-Jun N-terminal kinases (JNKs) and c-Jun, the involvement of JNKs in regenerative processes of the nigrostriatal pathway is controversially discussed. In our study, we utilized a mechanical scratch lesion paradigm of midbrain DAergic neurons in vitro and studied regenerative neuritic outgrowth. After a siRNAmediated knockdown of each of the three JNK isoforms, we found that JNKs differentially regulate neurite regeneration. Knockdown of JNK3 resulted in the most prominent neurite outgrowth impairment. This effect was attenuated again by plasmid overexpression of JNK3. We also evaluated cell survival of the affected neurons at the scratch border. JNK3 was found to be also relevant for survival of MDN which were lesioned by the scratch. Our data suggest that JNK isoforms are involved in differential regulation of
\end{abstract}

Lars Tönges and Veronique Planchamp contributed equally to this work.

L. Tönges $\cdot$ V. Planchamp · J.-C. Koch $•$ M. Bähr $\cdot$ P. Lingor $(\bowtie)$ Department of Neurology, University Medicine Göttingen,

R.-Koch-Str. 40,

37075 Göttingen, Germany

e-mail: plingor@gwdg.de

M. Bähr $\cdot$ P. Lingor

DFG Research Center for the Molecular Physiology of the Brain (CMPB),

Göttingen, Germany

T. Herdegen

Institute of Pharmacology,

Universitätsklinikum Schleswig Holstein,

Campus Kiel,

Kiel, Germany cell death and regeneration in MDN depending on their neurite integrity. JNK3 appears to be required for regeneration and survival in the case of an environment permissive for regeneration. Future therapeutic approaches for the DAergic system may thus require isoform specific targeting of these kinases.

Keywords JNK3 - Dopaminergic (DAergic) neuron · Regeneration · Parkinson's disease

\section{Introduction}

Parkinson's disease (PD) is the second most common aging-related neurodegenerative disorder and is characterized by progressive loss of dopaminergic (DAergic) neurons in the substantia nigra pars compacta $(\mathrm{SNpc})$ of the midbrain (Olanow and Tatton 1999). Several gene mutations have been linked to familial PD (Farrer 2006) but the etiology of sporadic PD, which accounts for $90 \%$ to $95 \%$ of all PD cases, is largely undefined. Current hypotheses attribute an increased risk for sporadic PD to misfolding and aggregation of proteins, mitochondrial dysfunction and oxidative stress. At the time of PD diagnosis, about $80 \%$ of DAergic neuron terminals in the striatum and $60 \%$ of DAergic neurons in the SNpc are already lost in PD brains (Dauer and Przedborski 2003). Today's medical therapies can only focus on symptomatic relief, and there is a great need to identify new therapeutic targets for improved clinical performance. As studies aiming to protect DAergic neurons in the SNpc have largely failed (Olanow et al. 2008; Lohle and Reichmann 2010) there is increasing interest in DAergic axonal targets which most likely play an earlier role in disease pathology (Braak et al. 2003; Duda 2003; Conforti et al. 2007; 
Schulz-Schaeffer 2010) and may be the substrate of regenerative approaches. For example, functional enhancement and protection of DAergic terminals could significantly improve clinical symptoms in animal models of Parkinson's disease (Chung et al. 2009).

The c-Jun N-terminal kinases (JNKs) are commonly known as stress-activated protein kinases because their activities typically increase in response to different cellular environmental stresses like osmotic stress, redox stress, or irradiation (Davis 2000; Weston and Davis 2002). Once activated by a stress response or by inflammatory cytokines they can also mediate cellular apoptosis (Kyriakis and Avruch 1996; Ip and Davis 1998) - a function that is particularly prominent in neurons and neuron-like cells (Xia et al. 1995; Watson et al. 1998).

Increasing in vitro and in vivo evidence suggests a role for JNK in the pathogenesis of DAergic neuron death in several Parkinson's disease models (Peng and Andersen 2003). As one example, the JNK pathway including its upstream kinase MKK4 is activated in the nigrostriatal system after 1-methyl-4-phenyl-1,2,3,6-tetrahydropyridine (MPTP) application in mice (Saporito et al. 2000; Willesen et al. 2002). Consequently, CEP-1347, a pharmacological inhibitor of upstream kinases of JNK, was able to protect from MPTP-induced JNK activation and nigrostriatal DAergic neuron loss (Saporito et al. 1999). These data are confirmed by later studies that in addition looked more closely at destruction of axonal TH-positive DAergic terminals in the striatum. Together with a protracted expression and phosphorylation of c-Jun in the SNpc after median forebrain bundle lesion Crocker et al. found a pronounced loss of TH-positive terminals in the striatum. Dominant-negative c-Jun did not only inhibit axotomyinduced dopamine neuron death and attenuate phosphorylation of c-Jun in nigral neurons but also delayed the degeneration of TH-positive DAergic terminals in the striatum (Crocker et al. 2001). Systemic application of the mixed lineage kinase inhibitor CEP11004 in the 6hydroxydopamine model of Parkinson's disease inhibited the loss of DA neurons in the SNpc and preserve THpositive striatal termini, too (Ganguly et al. 2004).

Whereas the aforementioned studies manipulated the entire JNK pathway (mainly due to the lack of specific pharmacological inhibitors) there is evidence that the three mammalian
JNK proteins (Gupta et al. 1996) play different roles in the context of cell survival and axon regeneration. Neuronal cell stress was found to specifically activate only JNK2 and 3 (Coffey et al. 2002). The function of these two genes seems to be closely related: in mice with single or double jnk2 and jnk3 null mutations only the double null mutation could protect from apoptosis in the intrastriatal 6-hydroxydopamine neurotoxin model (Ries et al. 2008). These results are in opposite to the lack of protection in the axonal compartment, where rather intense axonal degeneration in the jnk2 and jnk3 null mutations was observed. Similarly, the upstream blockade of the JNK pathway in the same animal model using an adeno-associated virus vector delivery of dominant-negative forms of dual leucine zipper kinase strongly inhibited apoptosis and enhanced long-term survival of DAergic neurons but did not protect their axons (Chen et al. 2008).

In order to further clarify the role of the three JNK proteins for axonal regeneration in DAergic neurons we performed a study of differential siRNA-mediated knockdown of JNK isoforms and evaluated neurite regeneration and DAergic survival in the scratch paradigm of mechanically transected primary neurons in culture (Knoferle et al. 2010). We identify JNK3 as the most important isoform regulating neurite outgrowth and survival after lesion.

\section{Materials and Methods}

siRNAs and Plasmids

siRNA targeting rat JNK1, JNK2, JNK3 and EGFP (GFP-22 siRNA) were purchased from Qiagen (Hilden, Germany). siRNA sequences are provided in Table 1.

\section{Primary Midbrain Neuron Culture}

Primary midbrain DAergic cultures were prepared according to previously published protocols (Knoferle et al. 2010). Briefly, the mesencephalon floor of embryonic day 14 Wistar rats was dissected and the meninges were removed. The dissected tissue pieces were collected in ice-cold CMF and centrifuged at 1,000 rpm for $4 \mathrm{~min}$. Trypsin $(750 \mu 1$,

Table 1 Sequences for JNK1, JNK2, JNK3, and EGFP siRNA

\begin{tabular}{llll}
\hline & siRNA sequence & Accession number & Target sequence (bp) \\
\hline JNK1 siRNA & 5' AGGCGAGAGATTTGTTATCTA 3' & XM_341399 & $1,003-1,023$ \\
JNK2 siRNA & 5' CTGGTGAAAGGTTGTGTGATA 3' & NM_017322 & $668-688$ \\
JNK3 siRNA & 5' TCCGTATGTGGTGACGAGATA 3' & NM_012806 & $912-932$ \\
EGFP siRNA & 5' CGGCAAGCTGACCCTGAAGTTCAT 3' & - & - \\
\hline
\end{tabular}


$0.25 \%$, Sigma) was added to the tissue pellet and after $15 \mathrm{~min}$ of incubation at $37^{\circ} \mathrm{C}$ was inactivated with $750 \mu \mathrm{l}$ cold FCS. Tissue fragments were gently triturated, the cell suspension was centrifuged at 1,000 rpm for $4 \mathrm{~min}$ and resuspended in culture medium.

For RNA interference studies, $4 \times 10^{6}$ cells were transfected with $0.3 \mu \mathrm{g}$ siRNA and/or $0.5 \mu \mathrm{g}$ plasmidic DNA using Amaxa Nucleofector (Amaxa, Cologne, Germany). Cells were then plated at a density of 500,000/well on 24well plates (Sarstedt, Nümbrecht, Germany) containing coverslips coated with poly-D-lysine and laminin.

For JNK inhibition studies, cells were plated at a density of $500,000 /$ well on 24-well plates (Sarstedt) directly after dissection. From day 3, cells were incubated with $5 \mu \mathrm{M}$ of the small molecule ATP-competitive JNK inhibitor SP600125 (anthra(1,9-cd)pyrazol-6(2 H)-one; CalbioChem, Darmstadt, Germany) or DMSO (AppliChem, Darmstadt, Germany) for 3 days before lysis/fixation (Bennett et al. 2001).

Cell cultures were maintained at $37^{\circ} \mathrm{C}$ in a $5 \% \mathrm{CO} 2$ humified atmosphere in DMEM-F12 (Invitrogen) supplemented with $2.5 \mathrm{mg} / \mathrm{ml}$ BSA (35\%), 0.9\% D-(+)-glucose solution (45\%), $2 \mathrm{mM}$ L-glutamine (PAA Laboratories, Pasching, Austria), $5 \mu \mathrm{g} / \mathrm{ml}$ insulin, 1:100 N1 medium supplement and 1:100 PSN antibiotic mixture (Invitrogen, Scotland, UK) for 4, 7, or 9 days. Medium was changed $24 \mathrm{~h}$ after cell dissection and subsequently every second day.

\section{Scratch Assay and Phase Contrast Imaging}

Three days following cell plating, cells were submitted to mechanical transection using a self-made $2 \mathrm{~mm}$ broad silicon rubber scratch device. Each coverslip was microscopically examined to ensure completeness of the scratch. Three days after mechanical transection (on day 6), cells were incubated in a climate chamber for live cell imaging $\left(37^{\circ} \mathrm{C}, 5 \% \mathrm{CO}_{2}\right)$ on a fluorescence inverted microscope (Axiovert, Zeiss, Oberkochen, Germany) equipped with a CCD camera and AxioVision software (Zeiss, Göttingen, Germany). Contrast phase photographs of three random visual fields per culture well were taken with a $20 \times$ objective.

Immunocytochemistry

For DAergic cell visualization, cells were fixed after live imaging on day 6 in PFA 4\% $10 \mathrm{~min}$ at room temperature (RT, $22^{\circ} \mathrm{C}$ ), permeabilized with $100 \%$ ice-cold acetone (AppliChem) $10 \mathrm{~min}$ at $-20^{\circ} \mathrm{C}$, washed twice with PBS and blocked with $10 \%$ normalized goat serum $10 \mathrm{~min}$ at RT. Probes were incubated with the primary antibodies (rat antityrosine hydroxylase 1:250 (Advanced Immunochemicals, Long Beach, CA, USA) overnight at $4^{\circ} \mathrm{C}$. Following 2 PBS washes, appropriate Cy3- or Cy2-labeled secondary antibodies (1:250, Dianova, Hamburg, Germany) were applied for $45 \mathrm{~min}$ at $37^{\circ} \mathrm{C}$. Cells were then nuclear counter-stained with DAPI (4,6-diamidino-2-phenylindole) (Sigma, Taufkirchen, Germany) and mounted in Mowiol (Hoechst, Frankfurt, Germany). Fluorescence was observed and recorded on a Zeiss Axioplan 2 fluorescence microscope equipped with a CCD camera and AxioVision software (Zeiss). For evaluation of DAergic regeneration and survival at the scratch site, pictures of the entire scratch site were taken with a $20 \times$ objective. For evaluation of DAergic survival within the culture, pictures of two random visual fields per culture well were taken with a $20 \times$ objective.

\section{Quantification of Regenerating Fibers}

Neurite length was evaluated semi-automatically using the axon tracing module of "Image J" (Free Java software provided by the National Institutes of Health, Bethesda, Maryland, USA). For evaluation of whole culture regeneration, the length of the 20 longest axons extending from the scratch site of each photomicrograph was quantified. Neurite length was defined as the neurite extension from neurite tip to scratch site. For evaluation of DAergic regeneration, length of all TH-labeled axons extending from the scratch site of each photomicrograph was quantified. The DAPI-labeled edge was used to identify the scratch site. Results are expressed in \% of EGFPtransfected cells. Experiments were performed at least in triplicate.

\section{Quantification of DAergic Neuron Survival}

For survival of DAergic neurons at the scratch site, the number of TH-labeled cells located in the area comprising the first $50 \mu \mathrm{m}$ next to the scratch site was quantified.

\section{Statistics}

All in vitro experiments were reproduced at least in triplicate. Differences between groups were considered statistically significant according to a one-way ANOVA followed by a parametric multiple comparison test (Dunnett's test). Significances were indicated with ${ }^{*} p<0.05 ;{ }^{* *} p<0.01 ;{ }^{* * *} p<0.001$, unless otherwise stated.

\section{Results}

Pharmacological Inhibition of All JNK Isoforms (1, 2, and 3) Impairs Neurite Regeneration of Primary DAergic Neurons

In order to evaluate the effect of a combined inhibition of all three JNK isoforms we took advantage of the 
availability of the pharmacological small molecule ATPcompetitive pan-JNK inhibitor SP600125. Primary DAergic neurons were cultured under standard conditions. After 3 days neurites were mechanically transected and SP600125 was applied at the same time. The evaluation of neurite outgrowth of DAergic neurons from the scratch border after another 3 days showed a significantly decreased relative outgrowth of JNK inhibitor treated total neurons $(56.2 \pm 8.8 \%)$ in comparison to non-inhibitor treated cells that were set to $100 \%(100 \pm 13.0 \%)$ (Fig. 1). The same holds true for a specific analysis of only DAergic neurons that show a decreased outgrowth after JNK inhibitor treatment $(64.4 \pm 32.8 \%)$ in comparison to untreated DAergic neurons $(100 \pm 15.9 \%)$. There was no significant difference between regeneration of DAergic and non-DAergic neurons.

siRNA-Mediated Downregulation of JNK3 Most Significantly Impairs Regenerative Neurite Outgrowth in Primary MDN

Midbrain DAergic neurons (MDN) cultures were separately transfected with siRNAs directed against JNK1, 2, and 3 . After 3 days in vitro, the neurons were scratch lesioned and their regenerative neurite outgrowth was evaluated after another 3 days (Fig. 2). In comparison to the siEGFP
Fig. 1 SP600125 significantly inhibits regenerative neurite outgrowth. a Representative micrographs of MDN cultures at 3 days after scratch lesion (upper panel, phase contrast; lower panel, TH-Cy2 (green) and DAPI (blue)). Cultures were treated with the pan-JNKinhibitor SP600125 $(5 \mu \mathrm{M})$ or DMSO control. Arrow, site of mechanical transection (scratch lesion). Scale bar, $150 \mu \mathrm{m}$. b Treatment with the pan-JNKinhibitor SP600125 (5 $\mu \mathrm{M})$ significantly impairs neurite regeneration of all primary cultured neurons including THpositive DAergic subpopulation after mechanical transection. Data are from three independent experiments each. Bars represent means $\pm \mathrm{SEM} ;{ }^{* * *} p<0.001$
A
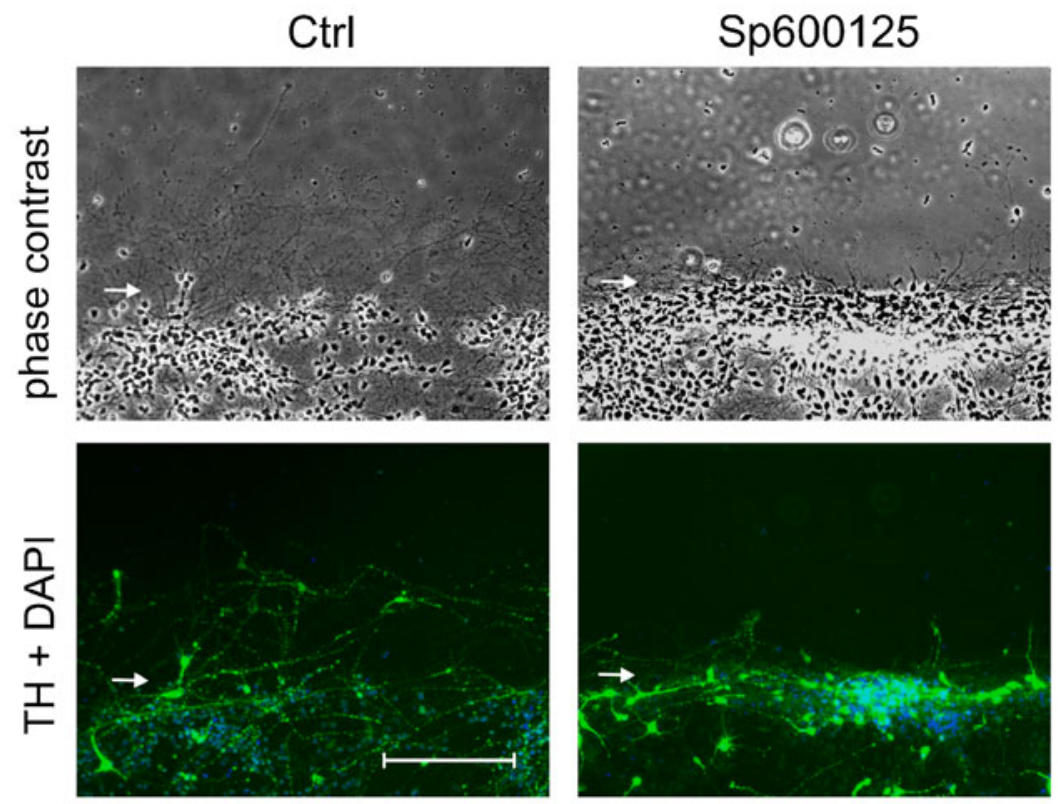

B

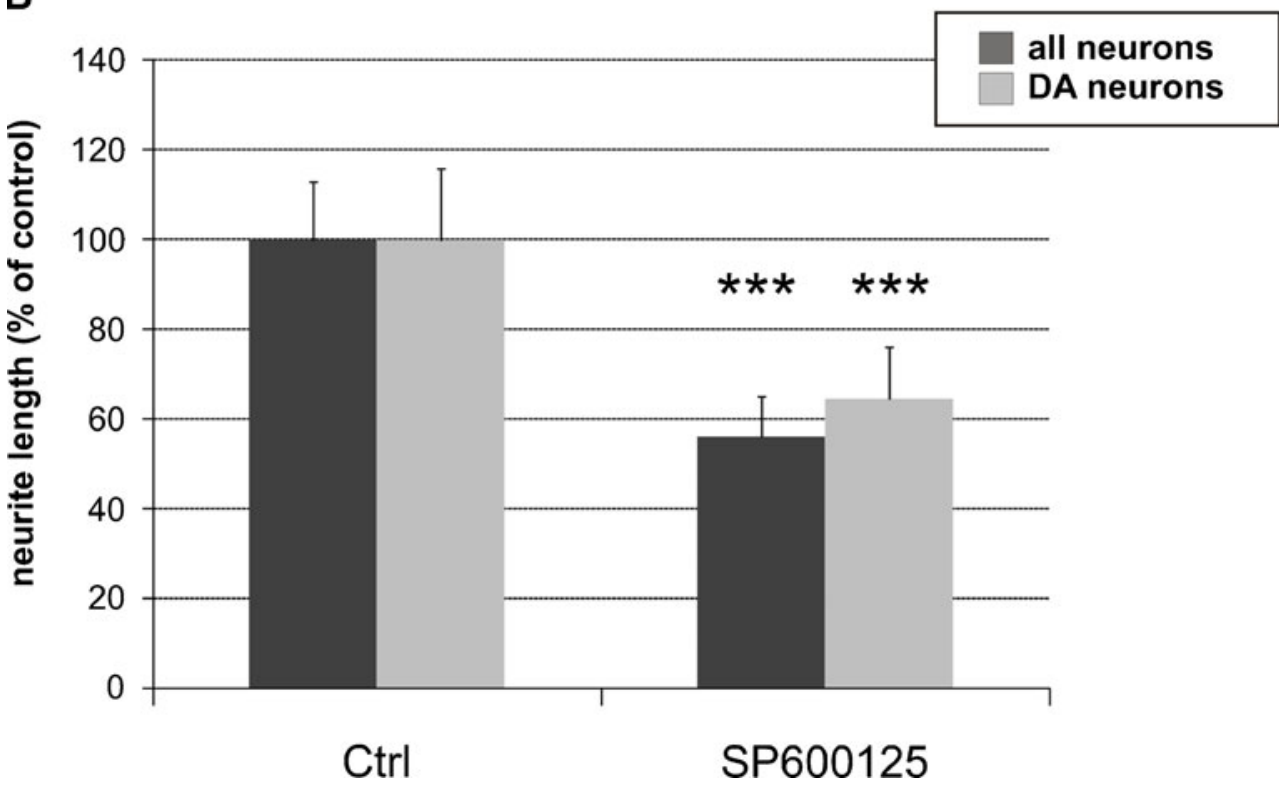


A

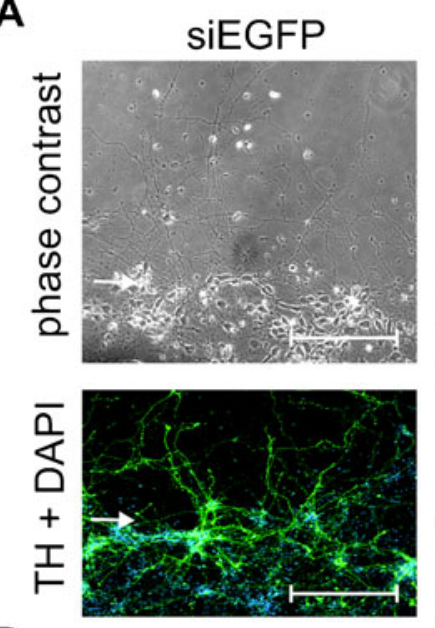

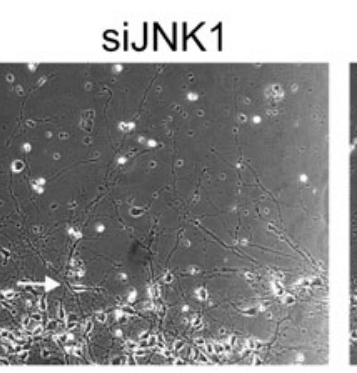




B



Fig. 2 Downregulation of JNK isoforms negatively influences regenerative neurite outgrowth. a Representative micrographs of MDN cultures at 3 days after scratch lesion (upper panel, phase contrast; lower panel, TH-Cy2 (green) and DAPI (blue)). Cultures were transfected with $0.3 \mu \mathrm{g}$ of siRNA directed against EGFP, JNK1, JNK2, or JNK3, respectively. Arrow, site of mechanical transection (scratch lesion). Scale bar, $200 \mu \mathrm{m}$. b Quantitative analysis of neurite

length at 3 days after mechanical transection in MDN cultures treated with different siRNAs for all neurons and for the DAergic subpopulation only. Application of JNK3 siRNA (siJNK3, $0.3 \mu \mathrm{g}$ ) resulted in the most significant decrease in neurite regeneration compared with cells transfected with EGFP siRNA (siEGFP) as a control. Bars represent means \pm SEM. For all treatments, $n=8 .{ }^{*} p<0.05 ; * * p<0.01$; $* * * p<0.001$

control $(100 \pm 11.4 \%)$, siJNK1 only slightly decreased neurite outgrowth in the total population $(85.6 \pm 10.2 \%)$. This effect was more pronounced with siJNK2, which reduced neurite outgrowth in the total population to $81.5 \pm$ $10.7 \%$ and in DAergic neurons to $90.3 \pm 13.1 \%$. Most significantly, knockdown of JNK3 reduced neurite outgrowth to $72.0 \pm 9.7 \%$ in the total population and to $86.2 \pm$ $14.4 \%$ in the DAergic neuron subpopulation.

siJNK3-Related Impaired Regenerative Response Can Be Efficiently Rescued by Overexpression of JNK3

As the neurite regenerative outgrowth was most dependent on the JNK3 isoform (Fig. 2), we overexpressed the JNK3 protein in culture to evaluate the specificity of our approach (Fig. 3). SiJNK3 transfection of MDN cultures resulted in a significantly impaired neurite outgrowth after scratch lesion compared with siEGFP transfection in the total population $(66.4 \pm 11.2 \%$ to $100.0 \pm 10.5 \%)$ and in the DAergic subpopulation $(70.5 \pm 11.9 \%$ to $100.0 \pm 16.9 \%)$ three days after lesioning. Expression of pEGFP (total population, $93.3 \pm 12.1 \%$; DAergic population, $122.8 \pm 22.5 \%$ ) or pJNK3 alone (total population, $101.8 \pm 13.7 \%$; DAergic population, $110.4 \pm 22.5 \%)$ did not significantly alter neurite outgrowth. However, the combined transfection of siJNK3 with pJNK3 significantly rescued the siJNK3 mediated impairment of neurite regeneration (total population, $94.5 \pm 13.8 \%$; DAergic population, $99.9 \pm 16.9 \%$ ). This effect could not be rescued by combined transfection of siJNK3 and pEGFP (total population, $74.7 \pm 12.5 \%$; DAergic population, $87.8 \pm 15.7 \%$ ). 
A
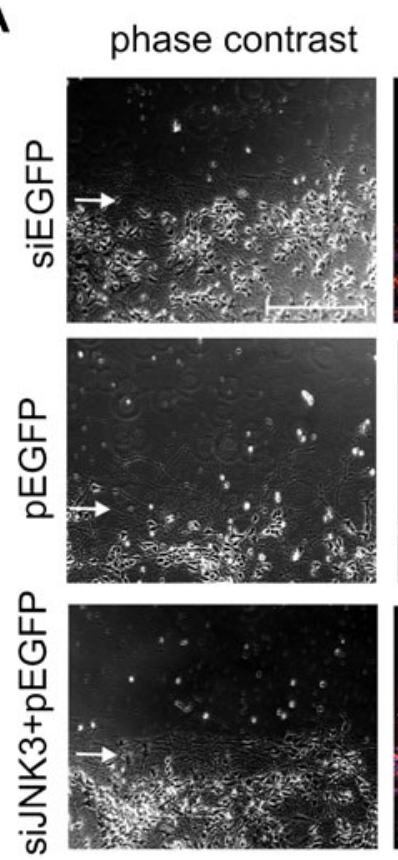

TH+DAPI
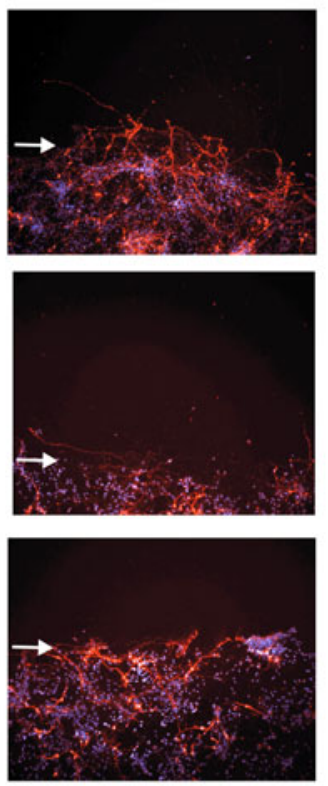

phase contrast


TH+DAPI
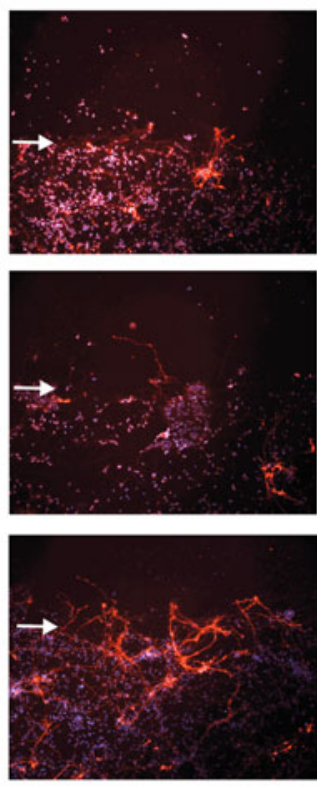

B

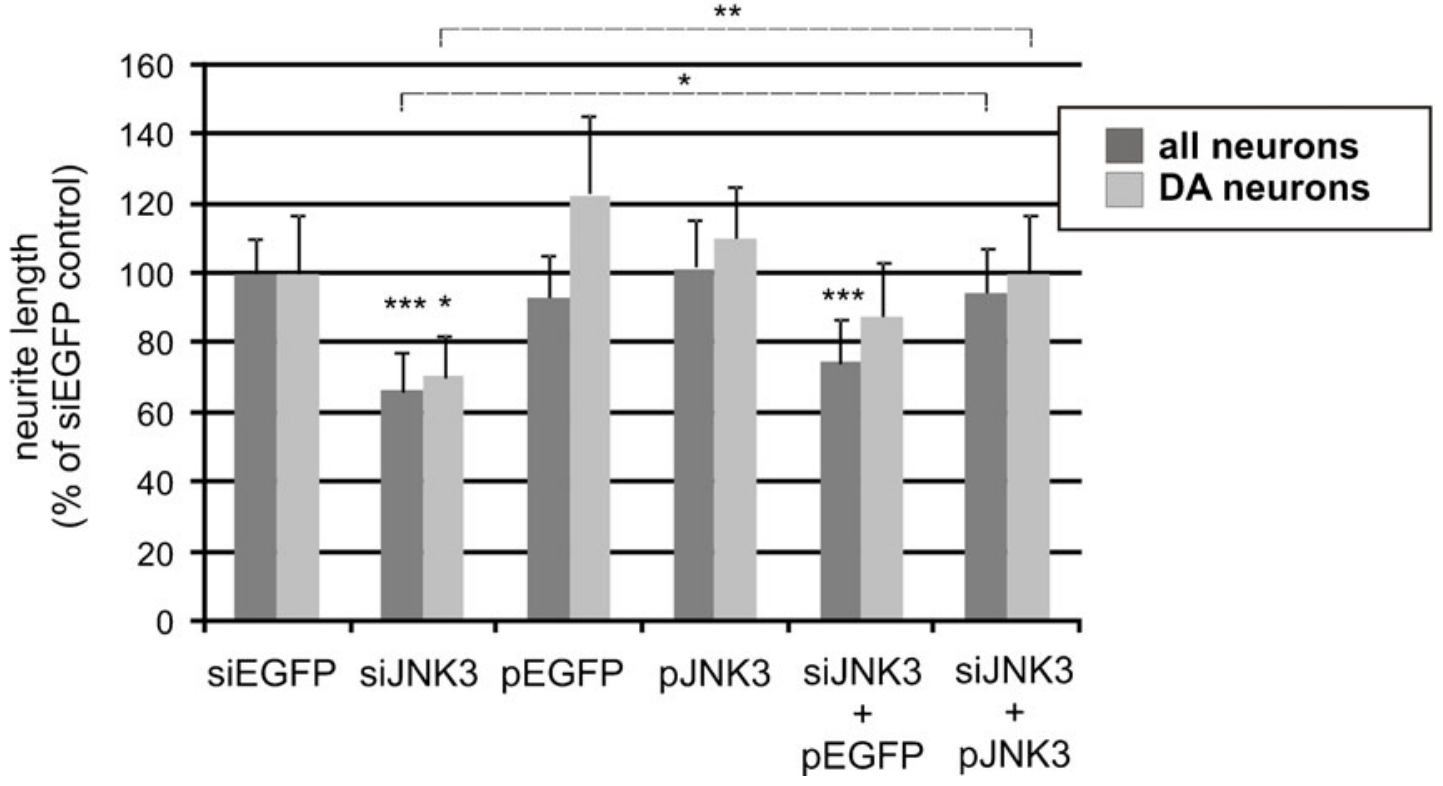

Fig. 3 JNK3 overexpression efficiently rescues the impaired regenerative response following JNK3 siRNA silencing. a Representative micrographs of MDN cultures at 3 days after scratch lesion (left panel, phase contrast; right panel, TH-Cy3 (red) and DAPI (blue)). Cultures were transfected on DIV0 with siRNA against EGFP or JNK3, the plasmids pEGFP or pJNK3, or a combination of JNK3 siRNA and pEGFP or JNK3 siRNA and pJNK3. Arrow, site of scratch lesion. Scale bar, $200 \mu \mathrm{m}$. b Quantitative analysis of neurite length at 3 days

Downregulation of JNK3 in Scratch Lesioned DAergic Neurons Impairs Survival

Apart from their role in neurite regeneneration JNK proteins play an important function in cell survival. Therefore, we evaluated the number of surviving TH- after scrach lesion in MDN cultures treated with siRNA or plasmid DNA as indicated. Neurite length is given for all neurons and for the DAergic subpopulation only. Overexpression of JNK3 rescues the siJNK3-mediated regeneration impairment, while transfection with pEGFP does not have a significant effect on neurite regeneration. Data are from three independent experiments each. Bars represent means \pm SEM. For all treatments, $n=8 .{ }^{*} p<0.05$; ${ }^{* *} p<0.01$; ${ }^{* * *} p<0.001$

positive neurons at the scratch border after 6 days in culture and siRNA transfection at DIV0 (Fig. 4). siJNK3 transfected neurons demonstrated a significantly reduced survival $(60.3 \pm 11.9 \%)$ compared with the siEGFP $(100.0 \pm$ $15.5 \%)$ as well as to the siJNK1 $(101.0 \pm 15.4 \%)$ and siJNK2 $(89.8 \pm 10.8 \%)$ transfected neurons. 
Fig. 4 JNK3 is important for survival of mechanically lesioned DAergic neurons. Transfection of $0.3 \mu \mathrm{g}$ of JNK3 siRNA significantly decreases the number of surviving DAergic neurons at the scratch border compared with EGFP-, JNK1and JNK2-siRNA-transfected neurons. For all treatments, $n=$ 8. Experiments were repeated at least three times with cells from different cultures. Bars represent means \pm SEM. ${ }^{* * *} p<0.001$

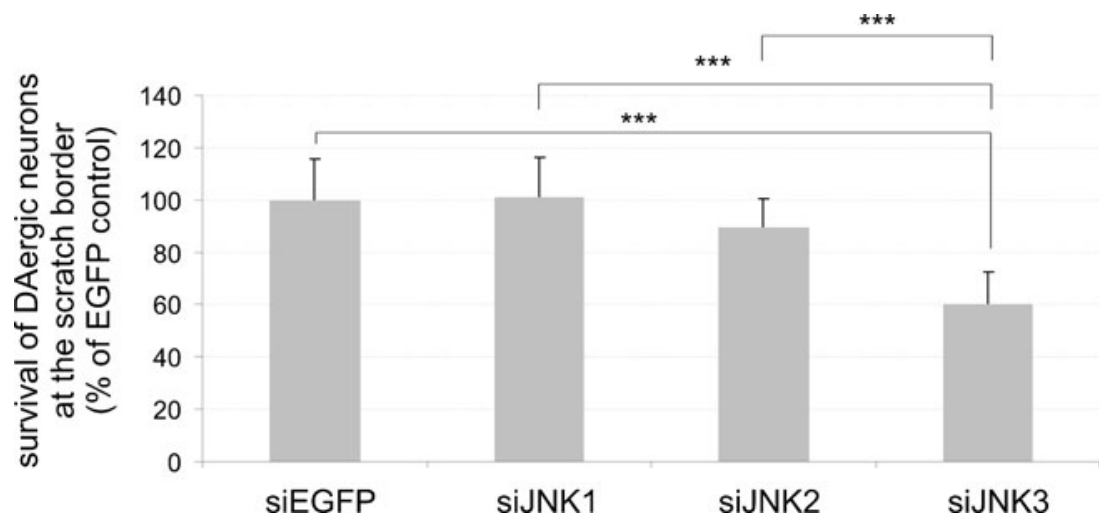

\section{Discussion}

In search for putative targets for neurorestorative strategies in Parkinson's disease (PD), we studied the role of JNK protein isoforms as they have been shown to participate in the pathophysiology of DAergic degeneration (Peng and Andersen 2003). We report here that the three JNK isoforms play distinct roles for neurite regeneration of DAergic neurons and that JNK3 seems to be the most relevant isoform in the regeneration paradigm after mechanical neurite lesion.

\section{Pharmacological Inhibition of JNK Negatively Affects} Neurite Regeneration

In the lesioned CNS, c-Jun phosphorylation occurs in axotomized neurons and correlates with enhanced longterm JNK activity (Herdegen et al. 1998). JNKs are able to trigger both expression and activation of c-Jun in lesioned neurons (Lindwall and Kanje 2005). However, several in vitro studies have shown that JNKs are also involved in neurite formation and stabilization (Leppa et al. 1998; Xiao and Liu 2003).

In our study, pan-JNK inhibition with SP600125 led to a severe impairment of neurite regeneration after scratch lesion in all neurons including the DAergic subpopulation (Fig. 1), which has been previously also reported for unlesioned MN9D cells and primary DAergic neurons (Eom et al. 2005). Thus, neurite growth in DAergic neurons depends on JNK activity in both primary and regenerative growth. Using RNAi technology we further investigated the question, which JNK isoform specifically regulates survival and regeneration in primary DAergic neurons.

\section{Differential Role of JNK1, 2, and 3 for Neurite}

Regeneration in Midbrain Neurons

In a previous study, we could demonstrate the importance of distinct protein kinase families for growth cone collapse, neurite retraction and neurite outgrowth (Loh et al. 2008).
We now show that the downregulation of all three JNK isoforms regulates the regenerative response in the total population, but only downregulation of JNK2 and even more JNK3 had significant effects on the DAergic subpopulation. Plasmid-mediated overexpression of JNK3 reversed the effect of single-time siRNA application and thus confirmed the importance of JNK3 in regenerative signaling. Interestingly, overexpression of JNK3 alone did not result in an increased regenerative growth, suggesting that JNK3 signaling is already saturated in this context.

JNK1 and JNK2 were previously shown to be involved in neurite outgrowth in PC12 cells (Waetzig and Herdegen 2003b). Whereas JNK1 and JNK2 are expressed in a large variety of tissues, the JNK3 isoform is restricted to brain, heart, and testis (Gupta et al. 1996). Although JNK3 was mainly attributed a pro-apoptotic function (Yang et al. 1997), it was also able to enhance NGF-induced sprouting in PC12 cells that normally do not express JNK3 (Waetzig and Herdegen 2003a). A putative explanation for JNK3associated neurite growth may be the fact that JNK3 together with JNK2 could be translocated to the nucleus and thereby influence transcription of regenerationassociated genes like c-Jun, ATF-3 (Lindwall et al. 2004), as well as CD44, galanin and a7b1 integrin (Raivich et al. 2004; Raivich and Makwana 2007). Depending on cell type and cellular context JNKs can bind and activate different binding partners in so-called signalosomes and thus, in principle, can trigger both degeneration and neurite outgrowth (Waetzig and Herdegen 2005; Waetzig et al. 2006). For example, in an analysis using knockout mice for jnkl, $j n k 2$, or jnk3, all animals presented a failure in neuritogenesis of hippocampal neurons, most prominently in the jnk3 knockout. However, this effect was not observed in cortical neurons (Eminel et al. 2008). Even more conflicting was the finding in cerebellar neurons, where JNKs relatively inhibited neuritogenesis (Coffey et al. 2000). Very recently, a study analyzing axonal regeneration in adult mouse dorsal root ganglion neurons demonstrated that neuritogenesis is delayed by lack of JNK2 and JNK3 but not by JNK1. JNK1 and JNK2 were rather necessary 
for sustained neurite elongation that was associated with the phosphorylation state of microtubule-associated protein MAP1b (Barnat et al. 2010). Our data support the notion that JNK3 is most prominently involved in regulation of the initial regenerative response in primary DAergic midbrain neurons.

JNK3 Is Relevant for Survival of Mechanically Lesioned DAergic Neurons

Our analysis demonstrated that JNK3-knockdown negatively affects survival of lesioned DAergic neurons at the scratch border (Fig. 4). Importantly, no general cell stressor was applied in this paradigm which may be the reason for the lack of detrimental signaling of JNK3. On the contrary, the regenerating and previously lesioned neurons seemed to partially depend on JNK3 since a downregulation resulted in less survival.

JNK3 appears to function as a key player in apoptosis (Waetzig and Herdegen 2003a). For example, jnk3 knockout mice lack excitotoxicity-induced apoptosis in the hippocampus (Yang et al. 1997). Targeted deletion of $j n k 3$ did not only reduce the stress-induced JNK activity, but also protected mice from cerebral ischemia-hypoxia that was attributed in part to a reduced expression of Bim and Fas (Kuan et al. 2003). Recently, the specific activation of the PI3K/Akt pathway has been identified as an additional neuroprotective mechanism by gene expression profiling in jnk3 knockout mice (Junyent et al. 2011). Novel data further extend the understanding of these processes by demonstrating that in principle all JNKs can induce cell death, which is critically dependent on nuclear localization and activation of cell death pathways (Bjorkblom et al. 2008)

In the context of PD the JNK/c-Jun pathway and especially the JNK3 isoform seem to play a leading role: in a human autopsy study c-Jun was found activated in DAergic neurons from PD patients-a result that was equally observed in the MPTP mouse model of PD (Hunot et al. 2004). In the same study, an examination of various JNK-deficient mice showed that both JNK2 and JNK3, but not JNK1, were required for MPTP-induced c-Jun activation and DAergic cell demise. Mice deficient for JNK3 showed also an enhanced and lasting survival of the MFB axotomized DAergic neurons in the SNpc (Brecht et al. 2005). JNK3 was also shown to mediate paraquat- and rotenone-induced DAergic neuron death, which could be attenuated by pharmacologically blocking translocation and activation of JNK3 (Choi et al. 2010). However, low JNK3 levels were not able to attenuate striatal dopamine terminal loss (Pan et al. 2009). In vivo studies in JNK3 or JNK2/3 knockout mice were not able to demonstrate axonal regenerative effects in a model of glaucoma (Quigley et al. 2011) or in an experimental model of PD (Cheng et al.
2010), which may be due to the more complex in vivo situation involving compensatory mechanisms in these genetically modified mice. Induced models to interfere with JNK3 signaling could possibly shed more light on the situation in vivo.

To this end, specific small molecule inhibitors of the JNK isoforms including JNK3 have been recently developed (Bowers et al. 2011; He et al. 2011; Probst et al. 2011; Schattel et al. 2011) and could be complementary to gene therapeutic approaches using siRNA/shRNA. Nevertheless, even with improved cell permeability of such JNK3 inhibitors, the achievement of a balanced specific spatial and temporal cellular inhibitory activity in vivo will still be required (Antoniou et al. 2011).

Studies on autopsy cases from patients treated with intraputaminal GDNF injections impressively demonstrate that regeneration can take place in PD (Love et al. 2005). Therefore, there is evidence to consider axonal regeneration as a therapeutic target mechanism in addition to neuroprotection in PD.

Taken together, we have shown that inhibition of JNK3 may result in a detrimental outcome regarding survival of previously lesioned and subsequently regenerating DAergic neurons. In our paradigm, JNK3 appears to be required for both axonal regeneration and survival. Thus, our results strongly support a pro-regenenerative role of JNK3 and attribute a pro-survival role specifically for lesioned dopaminergic neurons.

Acknowledgment We thank Elisabeth Barski for expert technical assistance.

Open Access This article is distributed under the terms of the Creative Commons Attribution Noncommercial License which permits any noncommercial use, distribution, and reproduction in any medium, provided the original author(s) and source are credited.

\section{References}

Antoniou X, Falconi M, Di Marino D, Borsello T (2011) JNK3 as a therapeutic target for neurodegenerative diseases. J Alzheimers Dis doi:10.3233/JAD-2011-091567

Barnat M, Enslen H, Propst F, Davis RJ, Soares S, Nothias F (2010) Distinct roles of c-Jun N-terminal kinase isoforms in neurite initiation and elongation during axonal regeneration. J Neurosci 30:7804-7816

Bennett BL, Sasaki DT, Murray BW, O'Leary EC, Sakata ST, Xu W, Leisten JC, Motiwala A, Pierce S, Satoh Y, Bhagwat SS, Manning AM, Anderson DW (2001) SP600125, an anthrapyrazolone inhibitor of Jun N-terminal kinase. Proc Natl Acad Sci USA 98:13681-13686

Bjorkblom B, Vainio JC, Hongisto V, Herdegen T, Courtney MJ, Coffey ET (2008) All JNKs can kill, but nuclear localization is critical for neuronal death. J Biol Chem 283:19704-19713

Bowers S, Truong AP, Neitz RJ, Neitzel M, Probst GD, Hom RK, Peterson B, Galemmo RA Jr, Konradi AW, Sham HL, Toth G, 
Pan H, Yao N, Artis DR, Brigham EF, Quinn KP, Sauer JM, Powell K, Ruslim L, Ren Z, Bard F, Yednock TA, GriswoldPrenner I (2011) Design and synthesis of a novel, orally active, brain penetrant, tri-substituted thiophene based JNK inhibitor. Bioorg Med Chem Lett 21:1838-1843

Braak H, Del Tredici K, Rub U, de Vos RA, Jansen Steur EN, Braak E (2003) Staging of brain pathology related to sporadic Parkinson's disease. Neurobiol Aging 24:197-211

Brecht S, Kirchhof R, Chromik A, Willesen M, Nicolaus T, Raivich G, Wessig J, Waetzig V, Goetz M, Claussen M, Pearse D, Kuan CY, Vaudano E, Behrens A, Wagner E, Flavell RA, Davis RJ, Herdegen T (2005) Specific pathophysiological functions of JNK isoforms in the brain. Eur J Neurosci 21:363-377

Chen X, Rzhetskaya M, Kareva T, Bland R, During MJ, Tank AW, Kholodilov N, Burke RE (2008) Antiapoptotic and trophic effects of dominant-negative forms of dual leucine zipper kinase in dopamine neurons of the substantia nigra in vivo. J Neurosci 28:672-680

Cheng HC, Ulane CM, Burke RE (2010) Clinical progression in Parkinson disease and the neurobiology of axons. Ann Neurol 67:715-725

Choi WS, Abel G, Klintworth H, Flavell RA, Xia Z (2010) JNK3 mediates paraquat- and rotenone-induced dopaminergic neuron death. J Neuropathol Exp Neurol 69:511-520

Chung CY, Koprich JB, Hallett PJ, Isacson O (2009) Functional enhancement and protection of dopaminergic terminals by RAB3B overexpression. Proc Natl Acad Sci USA 106:22474-22479

Coffey ET, Hongisto V, Dickens M, Davis RJ, Courtney MJ (2000) Dual roles for c-Jun N-terminal kinase in developmental and stress responses in cerebellar granule neurons. J Neurosci 20:7602-7613

Coffey ET, Smiciene G, Hongisto V, Cao J, Brecht S, Herdegen T, Courtney MJ (2002) c-Jun N-terminal protein kinase (JNK) 2/3 is specifically activated by stress, mediating c-Jun activation, in the presence of constitutive JNK1 activity in cerebellar neurons. J Neurosci 22:4335-4345

Conforti L, Adalbert R, Coleman MP (2007) Neuronal death: where does the end begin? Trends Neurosci 30:159-166

Crocker SJ, Lamba WR, Smith PD, Callaghan SM, Slack RS, Anisman H, Park DS (2001) c-Jun mediates axotomy-induced dopamine neuron death in vivo. Proc Natl Acad Sci USA 98:13385-13390

Dauer W, Przedborski S (2003) Parkinson's disease: mechanisms and models. Neuron 39:889-909

Davis RJ (2000) Signal transduction by the JNK group of MAP kinases. Cell 103:239-252

Duda JE (2003) Is the initial insult in Parkinson's disease and dementia with Lewy bodies a neuritic dystrophy? Ann NY Acad Sci 991:295-297

Eminel S, Roemer L, Waetzig V, Herdegen T (2008) c-Jun N-terminal kinases trigger both degeneration and neurite outgrowth in primary hippocampal and cortical neurons. J Neurochem 104:957-969

Eom DS, Choi WS, Ji S, Cho JW, Oh YJ (2005) Activation of c-Jun $\mathrm{N}$-terminal kinase is required for neurite outgrowth of dopaminergic neuronal cells. Neuroreport 16:823-828

Farrer MJ (2006) Genetics of Parkinson Disease: paradigm shifts and future prospects. Nat Rev Genet 7:306-318

Ganguly A, Oo TF, Rzhetskaya M, Pratt R, Yarygina O, Momoi T, Kholodilov N, Burke RE (2004) CEP11004, a novel inhibitor of the mixed lineage kinases, suppresses apoptotic death in dopamine neurons of the substantia nigra induced by 6hydroxydopamine. J Neurochem 88:469-480

Gupta S, Barrett T, Whitmarsh AJ, Cavanagh J, Sluss HK, Derijard B, Davis RJ (1996) Selective interaction of JNK protein kinase isoforms with transcription factors. EMBO J 15:2760-2770
He Y, Kamenecka TM, Shin Y, Song X, Jiang R, Noel R, Duckett D, Chen W, Ling YY, Cameron MD, Lin L, Khan S, Koenig M, Lograsso PV (2011) Synthesis and SAR of novel quinazolines as potent and brain-penetrant c-Jun N-terminal kinase (JNK) inhibitors. Bioorg Med Chem Lett 21:1719-1723

Herdegen T, Claret FX, Kallunki T, Martin-Villalba A, Winter C, Hunter T, Karin M (1998) Lasting N-terminal phosphorylation of c-Jun and activation of c-Jun N-terminal kinases after neuronal injury. J Neurosci 18:5124-5135

Hunot S, Vila M, Teismann P, Davis RJ, Hirsch EC, Przedborski S, Rakic P, Flavell RA (2004) JNK-mediated induction of cyclooxygenase 2 is required for neurodegeneration in a mouse model of Parkinson's disease. Proc Natl Acad Sci USA 101:665-670

Ip YT, Davis RJ (1998) Signal transduction by the c-Jun N-terminal kinase (JNK) - from inflammation to development. Curr Opin Cell Biol 10:205-219

Junyent F, de Lemos L, Verdaguer E, Folch J, Ferrer I, OrtunoSahagun D, Beas-Zarate C, Romero R, Pallas M, Auladell C, Camins A (2011) Gene expression profile in Jnk3 null mice: a novel specific activation of the Pi3k/Akt pathway. J Neurochem doi:10.1111/j.1471-4159.2011.07195.x

Knoferle J, Ramljak S, Koch JC, Tonges L, Asif AR, Michel U, Wouters FS, Heermann S, Krieglstein K, Zerr I, Bahr M, Lingor P (2010) TGF-beta 1 enhances neurite outgrowth via regulation of proteasome function and EFABP. Neurobiol Dis 38:395-404

Kuan CY, Whitmarsh AJ, Yang DD, Liao G, Schloemer AJ, Dong C, Bao J, Banasiak KJ, Haddad GG, Flavell RA, Davis RJ, Rakic P (2003) A critical role of neural-specific JNK3 for ischemic apoptosis. Proc Natl Acad Sci USA 100:15184-15189

Kyriakis JM, Avruch J (1996) Sounding the alarm: protein kinase cascades activated by stress and inflammation. J Biol Chem 271:24313-24316

Leppa S, Saffrich R, Ansorge W, Bohmann D (1998) Differential regulation of c-Jun by ERK and JNK during PC12 cell differentiation. EMBO J 17:4404-4413

Lindwall C, Kanje M (2005) The Janus role of c-Jun: cell death versus survival and regeneration of neonatal sympathetic and sensory neurons. Exp Neurol 196:184-194

Lindwall C, Dahlin L, Lundborg G, Kanje M (2004) Inhibition of cJun phosphorylation reduces axonal outgrowth of adult rat nodose ganglia and dorsal root ganglia sensory neurons. Mol Cell Neurosci 27:267-279

Loh SH, Francescut L, Lingor P, Bahr M, Nicotera P (2008) Identification of new kinase clusters required for neurite outgrowth and retraction by a loss-of-function RNA interference screen. Cell Death Differ 15:283-298

Lohle M, Reichmann H (2010) Clinical neuroprotection in Parkinson's disease-still waiting for the breakthrough. J Neurol Sci 289:104-114

Love S, Plaha P, Patel NK, Hotton GR, Brooks DJ, Gill SS (2005) Glial cell line-derived neurotrophic factor induces neuronal sprouting in human brain. Nat Med 11:703-704

Olanow CW, Tatton WG (1999) Etiology and pathogenesis of Parkinson's disease. Annu Rev Neurosci 22:123-144

Olanow CW, Kieburtz K, Schapira AH (2008) Why have we failed to achieve neuroprotection in Parkinson's disease? Ann Neurol 64 (Suppl 2):S101-S110

Pan J, Xiao Q, Sheng CY, Hong Z, Yang HQ, Wang G, Ding JQ, Chen SD (2009) Blockade of the translocation and activation of c-Jun $\mathrm{N}$-terminal kinase 3 (JNK3) attenuates dopaminergic neuronal damage in mouse model of Parkinson's disease. Neurochem Int 54:418-425

Peng J, Andersen JK (2003) The role of c-Jun N-terminal kinase (JNK) in Parkinson's disease. IUBMB Life 55:267-271

Probst GD, Bowers S, Sealy JM, Truong AP, Hom RK, Galemmo RA Jr, Konradi AW, Sham HL, Quincy DA, Pan H, Yao N, Lin M, 
Toth G, Artis DR, Zmolek W, Wong K, Qin A, Lorentzen C, Nakamura DF, Quinn KP, Sauer JM, Powell K, Ruslim L, Wright S, Chereau D, Ren Z, Anderson JP, Bard F, Yednock TA, Griswold-Prenner I (2011) Highly selective c-Jun N-terminal kinase (JNK) 2 and 3 inhibitors with in vitro CNS-like pharmacokinetic properties prevent neurodegeneration. Bioorg Med Chem Lett 21:315-319

Quigley HA, Cone FE, Gelman SE, Yang Z, Son JL, Oglesby EN, Pease ME, Zack DJ (2011) Lack of neuroprotection against experimental glaucoma in c-Jun N-terminal kinase 3 knockout mice. Exp Eye Res doi:10.1016/j.exer.2011.01.006

Raivich G, Makwana M (2007) The making of successful axonal regeneration: genes, molecules and signal transduction pathways. Brain Res Rev 53:287-311

Raivich G, Bohatschek M, Da Costa C, Iwata O, Galiano M, Hristova M, Nateri AS, Makwana M, Riera-Sans L, Wolfer DP, Lipp HP, Aguzzi A, Wagner EF, Behrens A (2004) The AP-1 transcription factor c-Jun is required for efficient axonal regeneration. Neuron 43:57-67

Ries V, Silva RM, Oo TF, Cheng HC, Rzhetskaya M, Kholodilov N, Flavell RA, Kuan CY, Rakic P, Burke RE (2008) JNK2 and JNK3 combined are essential for apoptosis in dopamine neurons of the substantia nigra, but are not required for axon degeneration. J Neurochem 107:1578-1588

Saporito MS, Brown EM, Miller MS, Carswell S (1999) CEP-1347/ KT-7515, an inhibitor of c-Jun N-terminal kinase activation, attenuates the 1-methyl-4-phenyl tetrahydropyridine-mediated loss of nigrostriatal dopaminergic neurons in vivo. J Pharmacol Exp Ther 288:421-427

Saporito MS, Thomas BA, Scott RW (2000) MPTP activates c-Jun NH (2)-terminal kinase (JNK) and its upstream regulatory kinase MKK4 in nigrostriatal neurons in vivo. J Neurochem 75:1200-1208

Schattel V, Hinselmann G, Jahn A, Zell A, Laufer S (2011) Modeling and benchmark data set for the inhibition of c-Jun N-terminal kinase-3. J Chem Inf Model doi:10.1021/ci100410h
Schulz-Schaeffer WJ (2010) The synaptic pathology of alphasynuclein aggregation in dementia with Lewy bodies, Parkinson's disease and Parkinson's disease dementia. Acta Neuropathol 120:131-143

Waetzig V, Herdegen T (2003a) A single c-Jun N-terminal kinase isoform (JNK3-p54) is an effector in both neuronal differentiation and cell death. J Biol Chem 278:567-572

Waetzig V, Herdegen T (2003b) The concerted signaling of ERK1/2 and JNKs is essential for PC12 cell neuritogenesis and converges at the level of target proteins. Mol Cell Neurosci 24:238-249

Waetzig V, Herdegen T (2005) Context-specific inhibition of JNKs: overcoming the dilemma of protection and damage. Trends Pharmacol Sci 26:455-461

Waetzig V, Zhao Y, Herdegen T (2006) The bright side of JNKsMultitalented mediators in neuronal sprouting, brain development and nerve fiber regeneration. Prog Neurobiol 80:84-97

Watson A, Eilers A, Lallemand D, Kyriakis J, Rubin LL, Ham J (1998) Phosphorylation of c-Jun is necessary for apoptosis induced by survival signal withdrawal in cerebellar granule neurons. J Neurosci 18:751-762

Weston CR, Davis RJ (2002) The JNK signal transduction pathway. Curr Opin Genet Dev 12:14-21

Willesen MG, Gammeltoft S, Vaudano E (2002) Activation of the cJun $\mathrm{N}$ terminal kinase pathway in an animal model of Parkinson's disease. Ann NY Acad Sci 973:237-240

Xia Z, Dickens M, Raingeaud J, Davis RJ, Greenberg ME (1995) Opposing effects of ERK and JNK-p38 MAP kinases on apoptosis. Science 270:1326-1331

Xiao J, Liu Y (2003) Differential roles of ERK and JNK in early and late stages of neuritogenesis: a study in a novel PC12 model system. J Neurochem 86:1516-1523

Yang DD, Kuan CY, Whitmarsh AJ, Rincon M, Zheng TS, Davis RJ, Rakic P, Flavell RA (1997) Absence of excitotoxicity-induced apoptosis in the hippocampus of mice lacking the Jnk3 gene. Nature 389:865-870 